\title{
Rotational Dynamics for Design of Bidirectional Feedback during Manual Interaction
}

\author{
Roderick Murray-Smith ${ }^{1,2}$ and Steven Strachan ${ }^{1}$ \\ ${ }^{1}$ Department of Computing Science \\ University of Glasgow \\ Glasgow, Scotland \\ rodedcs.gla.ac.uk \\ ${ }^{2}$ Hamilton Institute \\ National University of Ireland, Maynooth \\ Maynooth, Ireland \\ steven.strachan@nuim.ie
}

\begin{abstract}
Rotational dynamic system models can be used to enrich tightlycoupled embodied control of movement-sensitive mobile devices, and support a more bidirectional, negotiated style of interaction. This can provide a constructive, as well as informative, approach to the design of engaging, playful elements in interaction mechanisms. A simulated rotational spring system is used for natural eyes-free feedback in both the audio and haptic channels, and in a Mobile Spatial Interaction application, using twisting and tilting motions to drag and drop content, where users perceived the effect of varying the parameters of the simulated dynamic system.
\end{abstract}

\section{Introduction}

Inertial sensing is now widely available in millions of mobile phones, music players and in computer games such as the Nintendo Wii. Samsung's SCH-S310 gesture recognition phone, released in 2005, was the first which could recognise simple motion gestures, and SonyEricsson's W580i, W910i mobile phones allow you to shake to shuffle your music, or change tracks. Designing and refining interaction with such techniques is a relatively new area, and the techniques used so far have leant heavily on classical interaction design, such that most gesture recognition systems classify certain conditions or motions as virtual buttons, and provide feedback based on discrete, abstract movements. Such classical approaches to gesture recognition can be difficult to learn, and break down when subject to disturbances which are common in mobile environments. This paper presents a contrasting, physical modelling approach which enables the provision of continuous interaction and rich feedback during gesture-like interaction, which has advantages when learning a new interaction, or using the system in adverse conditions. Physical modelling is increasingly used in game design 1 for the virtual environment the player interacts with, providing increased levels of realism, and reducing development time, as the designer does not need to pre-specify every possible interaction. This also means that users can sometimes complete tasks in ways designers had not anticipated.

\footnotetext{
${ }^{1}$ e.g. the Havok physics engine www. havok.com

P. Markopoulos et al. (Eds.): Fun and Games 2008, LNCS 5294, pp. 1-10 2008.

(C) Springer-Verlag Berlin Heidelberg 2008
} 
The interaction metaphor proposed in this paper uses the simulation of a rotational dynamic system driven by inertial sensor data, to facilitate and enrich interaction with a mobile device. Such tilt-based interaction is well-established (Rekimoto 1996, Hinckley 2002, Hinckley et al. 2000), but typically depended primarily on visual feedback, which in tilting interactions causes difficulty due to poor screen visibility. Our intention in using a tangible physical metaphor for interaction is that users instantly possess a natural intuition for the effects that their movements have on the system. Our earlier work looked at dynamic models linking the zoom level to speed of motion in tilt-based interaction (Eslambolchilar and Murray-Smith 2004). Feedback is provided via audio and haptic rendering of the internal states of the simulated system. Which aspects of the state vector you choose to feedback, be it torque, velocity, friction or a combination, depends on the metaphor presented to the user. Allowing users to perceive the changing physical characteristics of the modelled system in this way can thus be used to convey richer information about the current state of their device. Another significant advantage of this approach is that the user experience may be easily tuned in realtime by simply adjusting the parameters of the modeled system as a function of variables of interest to the user, allowing the interaction to provide a bi-directional coupling of the user and computer. Sheridan (2002) provides a concise discussion of ways in which humans couple with their environments.

This approach can be applied to any tilt-based system as a way to enrich the interaction experience but as an example we introduce the simulation of a rotational spring system for twisting-based interaction with a mobile device and we show how basic aspects of this kind of system can be associated with information, and can be manipulated by a user in an intuitive fashion. This allows its use in a range of settings from games input to mobile spatial interaction. The same models can be used for pitch tilting, lateral roll actions, and for yawing actions (where the yaw sensing usually requires a magnetometer and/or gyroscope sensors, as well as accelerometers).

\section{Background}

Eyes-free interfaces rely heavily on the provision of effective audio and vibrotactile sensations. Yao and Hayward (2006) investigated the simulation of physical systems with audio and vibrotactile feedback, recreating the sensation of a ball rolling down a hollow tube via the haptic and audio modalities. Using apparatus that simulated the physics and provided audio and haptic cues, they found that when subjects were asked to estimate the position of the ball rolling inside a tubular cavity, they used their natural intuition of objects falling under the influence of gravity to accurately estimate the position. Similarly, Rath and Rocchesso (2005) created a convincing sonification of the physical motion of a ball along a beam, finding that subjects were able to perceive the ball motion from the sonification alone.

Shoogle (Williamson et al. 2007) enables the sensing of the state of a mobile device via the simulation of a physical system which responds to gestural input. By modelling the relatively simple dynamics of some balls inside a box and the quite intuitive effects of a users shaking of this box, information can be conveyed to the user such as the battery life of the device or number of new text messages, via auditory impact sounds 
and haptic rendering, where each new text message is represented by one simulated ball sensed only by the shaking of the device. (Hummels et al. 2007) used stroking interactions with tactile objects, with capacitive and force sensing. Early work on tapbased interaction in mobile phones includes (Linjama and Kaaresoja 2004).

\section{Rotational Spring Systems}

There is a wide range of physical systems that could be used as metaphors for interaction and rotational spring systems are just one example. This kind of system has a number of features that make it appropriate for interaction design. Sensing the orientation state of the device via accelerometers allows us to use movement of the device to control interaction. Twisting the device, sensed via changes in roll angle, or tilting, sensed via changes in the pitch angle can be easily sensed and used to provide eyesfree feedback about the state of the device to the user. Some rotational metaphors that we can simulate using the dynamics of this kind of system include winding a clock, opening a door knob, turning a key or opening a box, all completely natural everyday metaphors for which people have a natural intuition and which can enhance and enrich the process of interaction.

There are a number of basic conventions that we must consider when describing this kind of system. Figure 1 defines some of the basic notation. $\theta$ is defined as the angular displacement of the rotating disk with respect to some reference and is expressed in radians, $\omega$ is defined as the angular velocity of the disk in radians per second, $\alpha$ is defined as the angular acceleration in radians per second ${ }^{2}$ and $\tau$ is the torque, the rotational analogue of Force, in Newton-meters where $\tau=J \alpha$.

The four important characteristics of this kind of system from an interaction design perspective are torque, friction, stiffness and mass, which can be used to feedback device states to the user. Torque is important because it provides us with a measure for the amount of force present in the system. If this is fed back to the user in some way, via the audio or haptic channels, it can provide the user with a real sense of how the system is reacting to certain events or movements. Friction can significantly affect the

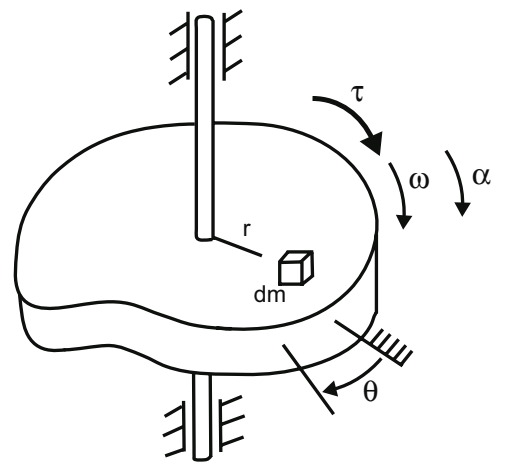

Fig. 1. Conventions for designating rotational variables. From (Close and Frederick 1995). 

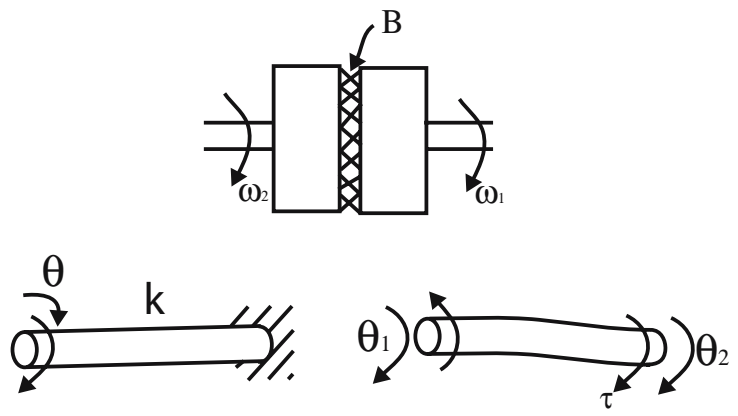

Fig. 2. a) Rotational stiffness element with one end fixed. b) Rotational stiffness element with $\Delta \theta=\theta_{2}-\theta_{1}$. From (Close and Frederick 1995).

feel of a rotational system. A rotational friction element is one for which there is an algebraic relationship between the torque and the relative angular velocity between two surfaces. Figure 2 a) shows rotational devices characterised by viscous friction, where the torque is defined as $\tau=B \omega$ (Close and Frederick 1995). So if we alter the friction between two surfaces the torque is altered and the state of the system can be fed back to and perceived by the user. Rotational stiffness is usually associated with a torsional spring, like that of a clock. An algebraic relationship between the torque $\tau$ and the angular displacement $\theta$ exists as illustrated in Figure $2 \mathrm{~b}$ ). For a linear torsional spring or flexible shaft $\tau=K \Delta \theta$ where $K$ is the spring constant. Altering the value of $K$ can then have an effect on the overall feel of the system. Higher $K$ values result in a more stiff system.

There are other kinds of mechanical system that could prove useful for this kind interaction design. Translational mechanics is one such example. When an object is moved on a surface it is possible to take a lot of information about the object and the surface from the sensation of the interaction between the two and varying degrees of friction between the object and surface are easily perceived via audio or haptic feedback.

\subsection{Two-Disk System}

It is possible then for us to view the mobile device as being a minimal inertia element, coupled with a rotational system via a rotational stiffness element as illustrated in figure 3. Angle changes in the orientation of the phone, sensed from accelerometers, act as reference values which drive the rotational system of interest, with the states of that system fed back to the user via vibration or audio. The systems we have chosen to simulate, the 'Two-Disk' system and the 'Disk and Mass' system are illustrated in Figures 3 and 5 respectively. We represent these systems using a state-space model similar to that described in (Eslambolchilar and Murray-Smith 2006). For the two-disk system we treat the angular displacement $\theta_{2}$ on disk 2 as an input to the system in order to observe the effects on $\theta_{1}$ and $\omega_{1}$ on disk 1 . This system can be represented as follows:

$$
\dot{x}=A x+B u
$$




$$
\left[\begin{array}{c}
\dot{\theta}_{1} \\
\dot{\omega}_{1}
\end{array}\right]=\left[\begin{array}{cc}
0 & 1 \\
-\frac{\left(k_{2}+k_{1}\right)}{J_{1}} & -\frac{B_{1}}{J_{1}}
\end{array}\right]\left[\begin{array}{c}
\theta_{1} \\
\omega_{1}
\end{array}\right]+\left[\begin{array}{c}
0 \\
\frac{k_{2}}{J_{1}}
\end{array}\right] \theta_{2}
$$

where $k_{1}$ and $k_{2}$ are the stiffness constants in shaft 1 and shaft 2 respectively, $B$ is the friction element for disk 1 and $J_{1}$ is the moment of inertia for disk 1 . If we imagine our mobile device to be represented by disk 2 and we exert some kind of roll-axis rotation on the device, this will induce a reaction in disk 1, the exact nature of which depends on the values chosen for $k_{1}, k_{2}$ and $B$. Figure 4 a) shows a typical response in the displacement angle $\theta$ for disk 1 from the two-disk system for varying values of $b_{1}$, the friction between disk 1 and the surface, after disk 2 has been twisted through $90^{\circ}$. As the friction parameter is increased, the simulated response of disk 1 to the input from disk 2 becomes increasingly damped. This more damped response, when fed back via the haptic and audio channels, can be clearly perceived by the user of the mobile device. Similar responses are observed for the varying of the $k_{1}$ and $k_{2}$ parameters of this model.

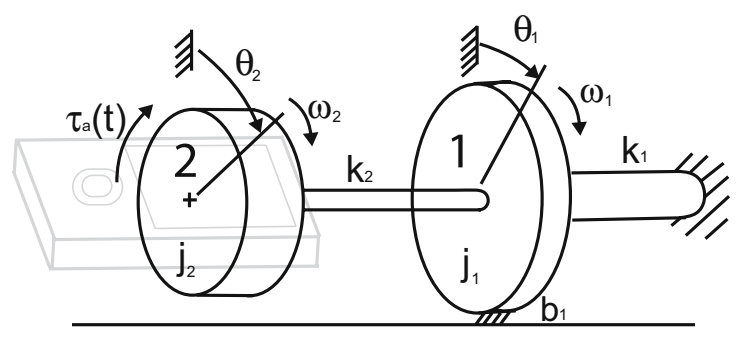

Fig. 3. Rotational system to illustrate the laws for reaction torques and angular displacements. Adapted from (Close and Frederick 1995).
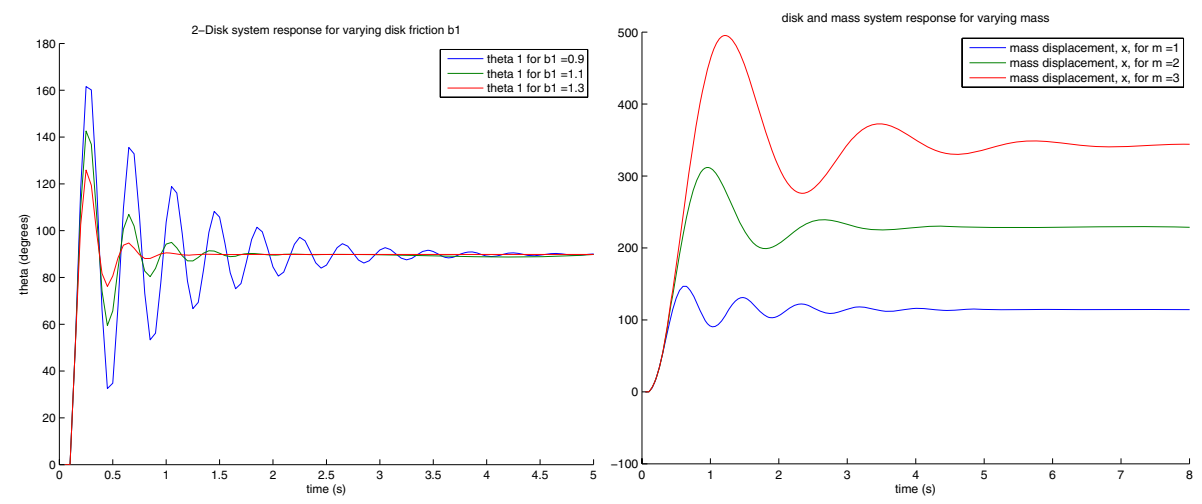

Fig. 4. a) System Response for a varying disk 1 friction $\left(b_{1}\right)$ in the Two-Disk system. As the friction is increased the simulated response of disk 1 decreases and this is perceived by the user. b) System Response for a varying mass parameter. As a mass attached to the spring is increased the simulated response becomes more extreme. 


\subsection{Disk and Mass System}

Figure 5 shows the same system but with a mass attached to the second disk via a spring, giving a sense of 'fishing' for content. There are a number of extra parameters for this system that can affect the way the system behaves. If the mobile device is rotated an effect is observed on disk 1 and hence on the movement of the mass, which also affects the torque experienced. Likewise, if we exert any force on the mass or change the value of the mass, this will also have an effect on the torque experienced in disk 1 . The exact effect observed depends on the states $x, v$ and $\theta_{1}, \theta_{2}$, measured from references corresponding to the position where the shafts $k_{1}$ and $k_{2}$ are not twisted and the spring $k_{3}$ is not stretched.

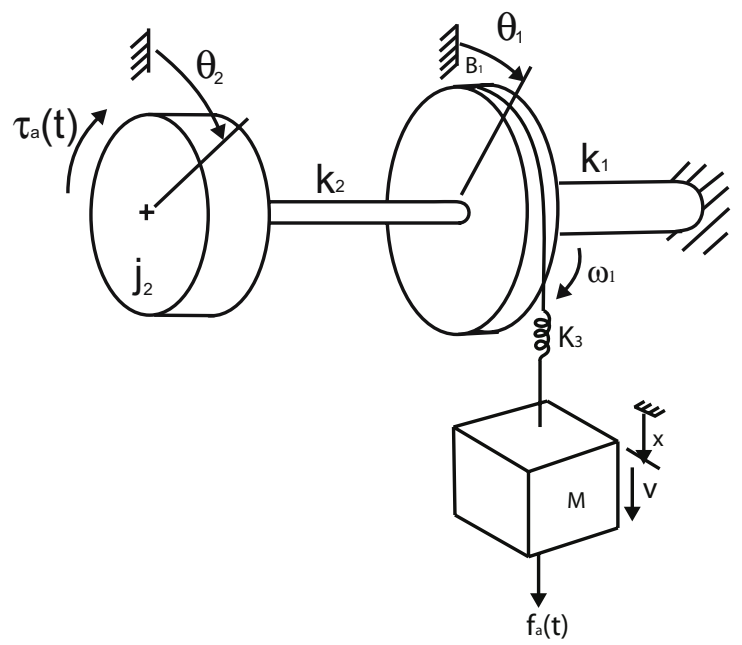

Fig. 5. System with translational and rotational elements. Adapted from (Close and Frederick 1995). This can be used to model drag-and-drop tasks, where the mass can be attached and detached.

Again, using a state-space approach we can represent the interaction dynamics of this system as follows:

$$
\left[\begin{array}{c}
\dot{\theta} \\
\dot{\omega} \\
\dot{x} \\
\dot{v}
\end{array}\right]=\left[\begin{array}{cccc}
0 & 1 & 0 & 0 \\
\frac{A R^{2}}{J_{1}} & -\frac{B_{1}}{J_{1}} & \frac{k_{3} R}{J_{1}} & 0 \\
0 & 0 & 0 & 1 \\
\frac{k_{3} R}{M} & 0 & -\frac{k_{3}}{M} & 0
\end{array}\right]\left[\begin{array}{l}
\theta \\
\omega \\
x \\
v
\end{array}\right]+\left[\begin{array}{cc}
0 & 0 \\
0 & \frac{k_{2}}{J_{1}} \\
0 & 0 \\
1 & 0
\end{array}\right]\left[\begin{array}{c}
m g \\
\theta_{2}
\end{array}\right]
$$

where $A=-k_{1}+k_{2}+k_{3}$. The inputs to this system are the external force on the mass $f_{a}(t)=m g$ and the displacement angle of disk 2, $\theta_{2}$. Varying the parameters of this model can significantly alter the feel of the interaction. For example, it is possible to convey the carrying of a varying mass by simply changing the mass parameter of the model, which then creates a different response. Figure $4 \mathrm{~b}$ ) shows how the system 
response changes as three different masses are added to the system. These varying responses, if converted to audio or vibrotactile feedback, are then perceived by the user. Similar responses are observed if we vary parameters $k_{1}, k_{2}$ and $k_{3}$.

\section{Mobile Spatial Interaction}

As an example use-case, we consider Mobile Spatial Interaction (MSI) - a form of interaction that enables users to interact in an eyes-free manner with a combined phys$\mathrm{ical} / \mathrm{virtual}$ environment using their mobile device to scan the space around them using a virtual probe to discover information as illustrated in figure 6. For background on bearing-based MSI, see (Strachan and Murray-Smith 2008, Frohlich et al. 2008). An example of a mobile game is (Ballagas et al. 2007). The direction in which the user is pointing is taken from the magnetic compass heading, with accelerometers used to infer the orientation (pitch and roll) of the device. This orientation is used to control how far into the distance the user is pointing. To look ahead, the device is tilted forward, to bring the probe back to the current position the device is tilted back. The user obtains information about the space around them by listening and feeling for impact events, when their probe effectively collides with targets or information in the augmented content in the virtual environment. It is also possible for users to manipulate and arrange objects in the virtual environment, picking up and moving them around using the device.

The disk and mass dynamics described enhance this interaction by providing the user with a sense of the varying kinds of information that they are interacting with. By twisting the device to pick up an object, users can immediately perceive the increasing spring tension as an object of information becomes an attached mass. Users can constantly monitor the state of their system just by twisting the device and perceiving the response of the dynamics. Is the system currently 'stiff', indicating the presence of a lot of local information or 'loose', indicating an absence of local information? Is there a heavy mass, light mass or no mass attached to the system? Feedback is provided via audio and haptic cues. Simply providing discrete pulses and sounds to indicate different states lacks the intuitiveness, subtlety and richness of an oscillating dynamic system
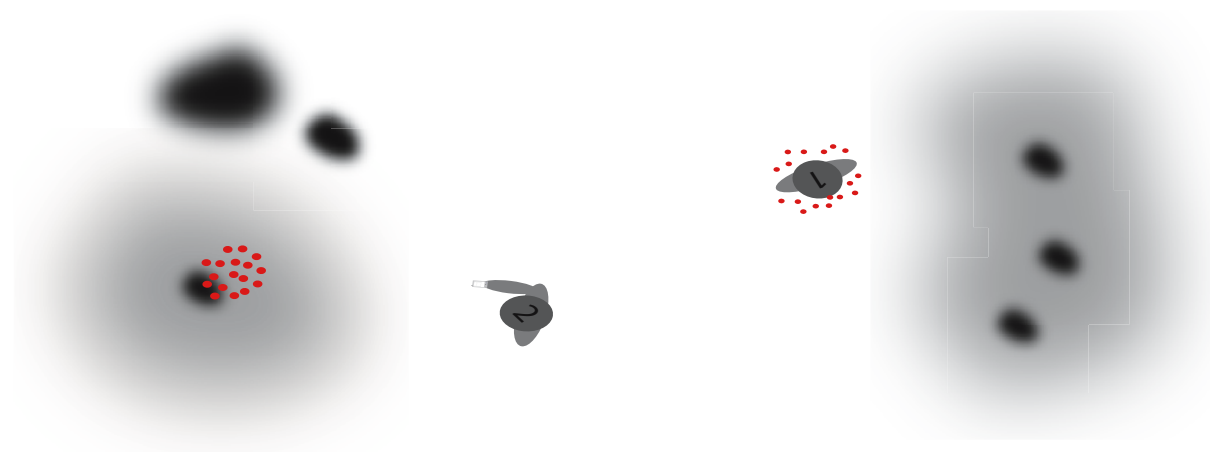

Fig. 6. A user interacting with information in their combined virtual/physical environment 


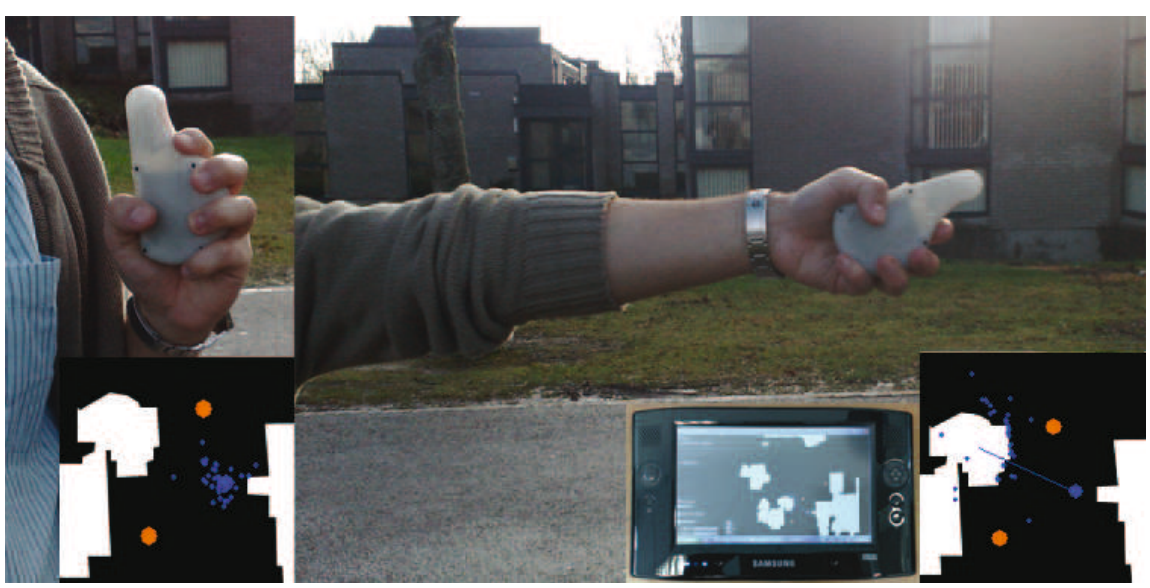

Fig. 7. A Samsung Q1 UMPC with a Bluetooth connection the WayStane inertial sensing device held in a users hand. Varying the orientation of the device alters the distance.

and can provide little information about the form or content of the object with which the user is interacting.

The current MSI system runs on a Samsung Q1 Ultra Mobile PC with a Bluetooth connection to the WayStane (Murray-Smith et al. 2008) inertial sensing device as shown in figure 7 This device, an adaptation of the SHAKE inertial sensing device (Williamson et al. 2007), contains the magnetometers, accelerometers and vibration devices required to produce this kind of interaction.

An initial user study was conducted where 5 participants were asked to comment on their perception of the system for varying parameters. First users were given a description of the Two-Disk system, with an illustrative figure (figure 3) and allowed to test it. The friction parameter $b_{1}$ was varied for five different values and users were asked to mark on a scale of 1-20 how they perceived the stiffness of the system as they applied $90^{\circ}$ twists to the device.

Participants were then given a description of the second system using figure 5 and allowed to test it. The value of the mass parameter was then varied (for five different values) as the mass was attached and released from the system in order to generate a reaction and the participants were again asked to comment on the perceived change in the system.

In the first part participants had little problem distinguishing a 'stiff' system from a 'loose' system. All participants were able to distinguish the five levels, with the only confusion coming from the more stiff settings. Subjective comments from participants indicated that it was easy to differentiate a stiff system from a loosely coupled system. In the second part, the participants were able to differentiate a very high mass from a low mass but values in between appeared to be more uncertain. The two participants who performed best in this part had the greatest knowledge of dynamic systems. The others had more trouble in interpreting the slightly more complicated system, and their subjective comments reflected this. Other comments included the fact that the 'kick back' from the system when the mass was released was slightly easier to perceive than 
the attaching of the mass itself. We expect that a refinement of the audio and tactile cues which are linked to the dynamic model states will improve some of the trouble participants had in intuitively understanding the more complex model.

\section{Outlook}

This paper has sketched the potential use of rotational dynamic systems for the provision of rich feedback to the user of a mobile device or remote control. By taking advantage of user's natural familiarity with the dynamics of a rotational spring mechanical system, as would be found in a door-handle, we have shown that it is possible to produce an eyes-free multimodal display using a solid theoretical foundation as the basis for the interaction. Coupling such rich continuous-feedback models with real-time inference about the content and relevance of information sources on the mobile device allows designers to make the user aware of subtle variations in the nature of the content they are engaging with and manipulating. It is an important building block for enabling negotiated interaction, where there is a bi-directional flow of information during interaction with interface objects.

This introductory paper only considers linear friction and spring models, but it is straightforward to expand the idea to richer models. The most interesting immediate area is that of non-linear friction models. Friction characteristics allow us to make the angular velocity 'visible' in the torque the user can sense, and also with appropriate torque/relative velocity characteristic curves we can create dead-zones in which an applied torque has no effect. We can create patterns of varying friction coefficients at different angles, to achieve specific desired effects. It is also possible to include other mechanical features such as gears or levers in the interaction design metaphors. For example, if gearing ratios were made audible the user might be able to perceive motion through different menu sizes, for example. In general, there is broad scope for multimodal displays which could, e.g. display the velocity data in audio, and the torque in vibration, and which could have richly nuanced variations in audio and tactile patterns as a function of the friction characteristics.

The applicability of the rotational spring metaphor to a Mobile Spatial Interaction application was demonstrated but the potential exists for a wider range of applications, such as personal music players, where the content drives the dynamics of the rotational systems as the user navigates the tracks, desktop-based drag and drop applications and eyes-free interaction. Our initial exploration suggests that users can perceive the variation in this mechanism, but that tuning the cues and feedback are important to maintain the illusion of the chosen metaphor. We suggest that research which couples rich content inference mechanisms with realistic dynamic models, and high quality, low-latency haptic feedback devices will lead to systems which are engaging, fun and easier to use.

Acknowledgements. We are grateful for support from: SFI grants 00/RFP06/CMS052, 00/PI.1/C067, EPSRC project EP/E042740/1, European Commission OpenInterface Project. Nokia provided a donation of funds and equipment. 


\section{References}

Ballagas, R.A., Kratz, S.G., Borchers, J., Yu, E., Walz, S.P., Fuhr, C.O., Hovestadt, L., Tann, M.: Rexplorer: a mobile, pervasive spell-casting game for tourists. In: CHI 2007: CHI 2007 extended abstracts on Human factors in computing systems, pp. 1929-1934. ACM, New York (2007)

Close, C., Frederick, D.: Modeling and Analysis of Dynamic Systems, 2nd edn. John Wiley and Sons, Chichester (1995)

Eslambolchilar, P., Murray-Smith, R.: Tilt-based automatic zooming and scaling in mobile devices - a state-space implementation. In: Brewster, S., Dunlop, M. (eds.) Mobile HCI 2004. LNCS, vol. 3160, pp. 120-131. Springer, Heidelberg (2004)

Eslambolchilar, P., Murray-Smith, R.: Model-based, multimodal interaction in document browsing. In: Renals, S., Bengio, S., Fiscus, J.G. (eds.) MLMI 2006. LNCS, vol. 4299, pp. 1-12. Springer, Heidelberg (2006)

Fröhlich, P., Baillie, L., Simon, R.: Realizing the vision of mobile spatial interaction. Interactions 15(1), 15-18 (2008)

Hinckley, K.: Input technologies and techniques. In: Handbook of Human-Computer Interaction, pp. 151-168. Lawerence Erlbaum Associates, Mahwah (2002)

Hinckley, K., Pierce, J., Sinclair, M., Horvitz, E.: Sensing techniques for mobile interaction. In: Proceedings User Interface Software and Technology (UIST 2000), pp. 91-100. ACM, New York (2000)

Hummels, C., Overbeeke, K.C., Klooster, S.: Move to get moved: a search for methods, tools and knowledge to design for expressive and rich movement-based interaction. Personal Ubiquitous Comput. 11(8), 677-690 (2007)

Linjama, J., Kaaresoja, T.: Novel, minimalist haptic gesture interaction for mobile devices. In: NordiCHI 2004: Proceedings of the third Nordic conference on Human-computer interaction, pp. 457-458. ACM Press, New York (2004)

Murray-Smith, R., Williamson, J., Quaade, T., Hughes, S.: Stane: synthesized surfaces for tactile input. In: CHI 2008: Proceeding of the twenty-sixth annual SIGCHI conference on Human factors in computing systems, pp. 1299-1302. ACM, New York (2008)

Rath, M., Rocchesso, D.: Continuous sonic feedback from a rolling ball. IEEE MultiMedia 12(2), 60-69 (2005)

Rekimoto, J.: Tilting operations for small screen interfaces. In: ACM Symposium on User Interface Software and Technology, pp. 167-168 (1996)

Sheridan, T.B.: Some musings on four ways humans couple: implications for systems design. IEEE Transactions on Systems, Man and Cybernetics, Part A 32(1), 5-10 (2002)

Strachan, S., Murray-Smith, R.: Bearing-based selection in mobile spatial interaction. Personal and Ubiquitous Computing (2008)

Williamson, J., Murray-Smith, R., Hughes, S.: Shoogle: excitatory multimodal interaction on mobile devices. In: CHI 2007: Proceedings of the SIGCHI conference on Human factors in computing systems, pp. 121-124. ACM, New York (2007)

Yao, H.-Y., Hayward, V.: An experiment on length perception with a virtual rolling stone. In: Eurohaptics 2006 (2006) 